\title{
ABSTRACTS OF POSTER PRESENTATIONS
}

\section{PA-001 OCCURRENCE OF DAY 3 SUBMICROSCOPIC PLASMODIUM FALCIPARUM PARASITAEMIA BEFORE AND AFTER IMPLEMENTATION OF ARTEMETHER-LUMEFANTRINE TREATMENT POLICY IN TANZANIA}

Billy Ngasala, ${ }^{1}$ |rina Jovel, ${ }^{2}$ Maja Malmberg, ${ }^{3}$ Bruno Mmbando, ${ }^{4}$ Anders Björkman, ${ }^{2}$ Zul Premji, ${ }^{5}$ Andreas Mårtensson, ${ }^{6}$ Richard Mwaiswelo ${ }^{1}$. 'MUHAS, Tanzania; ${ }^{2} \mathrm{Kl}$, Sweden; ${ }^{3}$ SLU, Sweden; ${ }^{4}$ NIMR, Tanzania; ${ }^{5}$ Aga Khan University Hospital, Kenya; ${ }^{6}$ Uppsala University, Sweden

\subsection{6/bmjgh-2016-000260.40}

Background Emergence of Plasmodium falciparum resistance against artemisinin in Southeast Asia raises a serious concern about the long-term efficacy of artemisinin-based combination therapy (ACT) globally. In Africa, ACT has remained highly efficacious with a microscopy determined asexual parasites clearance occurring within 48 hours post-treatment in most patients. However, submicroscopic parasitaemia has been reported on Day 3 after ACT treatment. We assessed the prevalence of patients with submicroscopic parasitaemia on Day 3 and its associated factors following treatment with artemether-lumefantrine (AL) from 2006 to 2014 in Bagamoyo district, Tanzania.

Methods Cytochrome b-nested polymerase chain reaction (PCR) was used for screening of submicroscopic parasitaemia from blood samples collected on filter paper on Day 3 post-AL treatment for acute uncomplicated P. falciparum malaria.

Primary outcome was proportion of patients with submicroscopic parasitaemia on Day 3 from 2006 to 2014. Secondary outcomes included proportional difference in submicroscopic parasitaemia across years, association of pre-treatment characteristics with submicroscopic parasitaemia, and association of submicroscopic parasitaemia with recurrent infection. 
Results Only 2/584 (0.34\%) of the screened patients had microscopy determined parasitaemia on Day 3, whereas, 256/ $584(43.8 \%)$ had submicroscopic parasitaemia. Submicroscopic parasitaemia prevalence increased from 28\% (14/50) in 2006 to $74.2 \%(132 / 178)$ in $2007-8$, and thereafter declined to $36 \%$ (50/139) in 2012-13 and $27.6 \%(60 / 217)$ in 2014, with the likelihood of being positive for submicroscopic parasitaemia decreasing by 14.7\% (95\% CI: 9.5-19.7\%, p<0.001) for an increase in year by one. Pre-treatment parasitaemia $>100,000 /$ $\mu \mathrm{L}$, haemoglobin $<10 \mathrm{~g} / \mathrm{dL}$, fever, being aged $<5$ years and year of study $2007-8$ and 2012-13 were associated with the presence of submicroscopic parasitaemia. There was no association between submicroscopic parasitaemia and recurrent infection.

Conclusions Day 3 submicroscopic parasitaemia was common in patients treated with AL before and after implementation of the policy, and changed considerably across years from 2006 to 2014, however, its presence was associated with pre-treatment characteristics. 